\title{
The amyloid cascade is not the only pathway to AD
}

\author{
Gaël Chételat
}

In my original News \& Views article (Alzheimer disease: $A \beta$-independent processes-rethinking preclinical AD. Nat. Rev. Neurol. 9, 123-124; 2013), ${ }^{1}$ I discussed contradictions between the conclusion made by Knopman et al. ${ }^{2}-$ namely, that the initial appearance of brain-injury biomarkers in preclinical Alzheimer disease (AD) may not depend on $\beta$-amyloidosis-and the current dominant view of the sequence of biomarkers in AD. Following a letter from V. Y. Vishnu (Can tauopathy shake the amyloid cascade hypothesis? Nat. Rev. Neurol. doi:10.1038/ nrneurol.2013.21-c1 $)^{3}$ in which he mentions a paper by Jack et al. ${ }^{4}$ that revisits the biomarker model, I would like to make some comments and clarifications.

Jack et al. ${ }^{4}$ insist on the distinction between the neuropathological processes and the biomarkers, which I agree is important. Knopman et al. ${ }^{2}$ suggest that, from a neuroimaging-based-biomarker perspective, neurodegeneration can appear before amyloid- $\beta(\mathrm{A} \beta)$ deposition. However, these biomarkers represent only the visible part of the AD 'iceberg'; inferences regarding the 'immersed' part (that is, the physiopathological mechanisms) should be made cautiously. Such inferences are supported by autopsy studies, ${ }^{5}$ leading Jack and colleagues ${ }^{4}$ to propose a model in which tauopathy is the first pathophysiological process in $\mathrm{AD}$. However, Jack et al. ${ }^{4}$ consider that, on neuroimaging, $A \beta$ deposition is always visible before tau-mediated neuronal injury. I argue for a more flexible scenario in which tau-related injury can appear before $\mathrm{A} \beta$, even at the biomarker level.

Another important point mentioned by Jack et al. ${ }^{4}$ is the distinction between sporadic versus genetically determined early-onset AD. Different processes and sequences may be involved in these two forms of the disease, and my comments mainly addressed the sporadic form.

Vishnu suggests that challenging the amyloid hypothesis is premature, concluding that " $\mathrm{A} \beta$ - either as initiator or as an accelerator of disease-is still the main factor in the pathogenesis of AD." ${ }^{3}$ The fact that $A \beta$ can accelerate-not always initiate-the pathological processes suggests that the amyloid cascade is not the only possible pathway to $\mathrm{AD}$. Consideration of an alternative pathway need not imply, however, that tauopathy is itself the initiator of the pathological cascade. Figure 1 of my original article ${ }^{1}$ depicts $A \beta$ and tau in parallel, with partly independent accumulation, and shows their interaction at the pathological level. I propose that expression of tau and $\mathrm{A} \beta$ be considered as equal only from a neuroimaging-biomarker perspective, without taking into account the sequence in which they appear.

$\mathrm{A} \beta$ and tau certainly have different roles in $\mathrm{AD}$ pathogenesis: tauopathy may be the weapon of neurodegeneration, and $\mathrm{A} \beta \mathrm{a}$ a booster and/or a guide for tauopathy.
Which factor is to blame? This question is important for therapeutic developments as the answer will dictate which factor(s) we should eliminate. The chronology of events also has implications from both biomarker and therapeutic perspectives: the fact that neurodegeneration is not necessarily dependent on, or resultant from, $A \beta$ should be considered. More and more evidence from both neuroimaging and neuropathological studies suggests that the amyloid cascade is not the only way to tau-related neurodegeneration and sporadic AD.

INSERM, U1077, CHU Avenue de la Côte de Nacre, CS 30001, 14033 Caen Cedex 9, France.

chetelat@cyceron.fr

Competing interests

The author declares no competing interests.

1. Chételat, G. Alzheimer disease: $A \beta$-independent processes-rethinking preclinical AD. Nat. Rev. Neurol. 9, 123-124 (2013).

2. Knopman, D. S. et al. Neuronal injury biomarkers are not dependent on $\beta$-amyloid in normal elderly. Ann. Neurol. http://dx.doi.org/ 10.1002/ana.23816.

3. Vishnu, V. Y. Can tauopathy shake the amyloid cascade hypothesis? Nat. Rev. Neurol. http:// dx.doi.org/10.1038/nrneurol.2013.21-c1.

4. Jack, C. R. Jr et al. Tracking the pathophysiological processes in Alzheimer's disease: an updated hypothetical model of dynamic biomarkers. Lancet Neurol. 12, 207-216 (2013).

5. Braak, H. \& Braak, E. Frequency of stages of Alzheimer-related lesions in different age categories. Neurobiol. Aging 18, 351-357 (1997). 